\title{
Comportamento germinativo de sementes de diferentes cores de pariparoba [Pothomorphe umbellata (L.) Miq.]
}

\author{
MAIA-ALMEIDA, C.I. ${ }^{1 *}$; CAVARIANI, C. ${ }^{2}$; OLIVEIRA, P.F.C. ${ }^{1}$; MING, L.C. ${ }^{1}$; MATTANA, R.S. ${ }^{1}$; LIMA, L.P. ${ }^{1}$ \\ ${ }^{1}$ Departamento de Produção Vegetal, Setor de Horticultura ${ }^{2}$ Setor de Agricultura e Melhoramento Vegetal, \\ Universidade Estadual Paulista-UNESP, Faculdade de Ciências Agronômicas, Rua José Antônio de Barros, 1780, \\ Caixa Postal 273, CEP: 18610-307, Botucatu-Brasil *iezidbr@yahoo.com.br
}

\begin{abstract}
RESUMO: A Pothomorphe umbellata (Piperaceae), é uma espécie medicinal nativa do Brasil, utilizada na indústria de cosméticos e protetores de pele contra raios UVA e UVB. Com o intuito de gerar informações aplicadas à propagação da espécie, o presente trabalho relacionou a coloração e a massa de sementes de $P$. umbellata a seu comportamento germinativo. A coloração e a massa de sementes de $P$. umbellata foram características adequadas para avaliar a homogeneidade fisiológica, o vigor, o potencial e o comportamento germinativo. Assim, concluise que, embora de germinação lenta, as sementes de coloração preta e mais densa devem ser as escolhidas quando de coleta ou de processo seletivo.
\end{abstract}

Palavras-chave: pariparoba, coloração do tegumento, massa de semente, Mata Atlântica, plantas medicinais

\begin{abstract}
Germinative behavior of Pothomorphe umbellata (L.) Miq. seeds of different colors. Pothomorphe umbellata (Piperaceae) is a medicinal species native to Brazil, which has been used in the cosmetic industry and in products that protect the skin against UVA and UVB rays. To generate information applied to the species propagation, the present work related the coloration and the mass of $P$. umbellata seeds to their germinative behavior. The coloration and the mass of seeds of $P$. umbellata were characteristic appropriate to evaluate physiologic homogeneity, vigor, potential and germinative behavior. Thus, although of slow germination, seeds of black and denser coloration should be chosen during collection or selective process.
\end{abstract}

Key words: pariparoba, tegument coloration, seed mass, Atlantic Forest, medicinal plants

\section{INTRODUÇÃO}

Pothomorphe umbelata (L.) Miq. (Piperaceae) é uma espécie medicinal nativa do Brasil, popularmente conhecida como pariparoba ou caapeba, utilizada na indústria de cosméticos para produção de dermoprotetores contra raios UVA e UVB e de antioxidantes, devido à presença do 4nerolidilcatecol. A obtenção da matéria prima é restrita ao extrativismo e às pequenas áreas de cultivo sob conhecimento agronômico científico ainda incipiente.

A compreensão e o manejo correto dos padrões fisiológicos da germinação permitem, até certo ponto, a expressão do potencial produtivo da espécie alvo na medida em que pode proporcionar adequada emergência de plântulas e uniformidade de população de plantas em campo. Assim, conseqüentemente, serão alcançadas melhorias na produção de mudas e no estabelecimento de estande em semeadura direta. Para espécies nativas medicinais, informações com este escopo são escassas proporcionando dificuldades ao manejo agronômico para à propagação por sementes.

$O$ estudo da germinação de sementes de espécies neotrópicas é fundamental para a conservação e o melhoramento genético na busca da redução do risco de extinção, além da viabilização econômica da espécie pelo manejo agronômico (Labouriau, 1983). O comportamento germinativo é produto da história da planta-mãe, dimorfismo ou polimorfismo das sementes e características da espécie (Maluf \& Wizentier, 1998; Larcher, 2000; Marcos Filho, 2005).

Trabalhos realizados em várias áreas da produção vegetal com diversas espécies, como alface, cebola, Phyllanthus, gervão roxo, Qualea grandiflora

Recebido para publicação em 27/02/2008

Aceito para publicação em 13/09/2010

Rev. Bras. Pl. Med., Botucatu, v.13, n.1, p.116-120, 2011. 
e Sebastiana commersoniana destacaram estreita correlação entre coloração do tegumento, tamanho e massa de sementes com qualidade fisiológica (germinação, vigor e sanidade) bem como à emergência de plântulas e desenvolvimento e produtividade à campo, (Felippe, 1990; Piana et al.,1995; Unander et al., 1995; Rosseto et al., 1997; Ventura \& Randi, 1997; Marcos Filho,1998; Marcos Filho, 1999; Rosseto et al. 2000; Rodo, 2002; Franzin et al., 2005; Santos \& Aguiar, 2005; Marcos Filho, 2005). Assim, o trabalho teve como objetivo relacionar a coloração e a massa de sementes de $P$. umbellata ao comportamento germinativo.

\section{MATERIAL E MÉTODO}

As sementes foram colhidas em 20 de março de 2006 em uma população nativa no município de Cajamar-SP, localizada a 2319,500، Sul e 46 53,335' Oeste e à $857 \mathrm{~m}$ de altitude, a partir de espigas maduras coletadas de 80 indivíduos vegetais distintos. Foram consideradas maduras as espigas cujo terço médio tinham sementes de coloração escura (marrons e pretas) e textura mucilaginosa.

Para obtenção das sementes as espigas colocadas em água destilada e maceradas com auxílio das mãos até a separação completa das partes. Após esta etapa, as sementes foram separadas por seis lavagens em água destilada permanecendo ao fundo do recipiente apenas as sementes. Para secagem parcial as sementes foram

$$
\begin{aligned}
& \operatorname{PG}(\%)=\left(\frac{\Sigma n i}{\mathrm{~N}}\right) * 100 \\
& \operatorname{CVG}(\%)=\left(\frac{\Sigma n i}{\Sigma n i * x i}\right) * 100 \\
& f_{i}=\frac{n i}{-\Sigma_{i=1}^{\mathrm{K}} n i}
\end{aligned}
$$

colocadas sobre papel toalha, por 15 dias em condições de ambiente natural $\left(20-25^{\circ} \mathrm{C}\right.$ e $60-70 \%$ de umidade relativa) sobre bancada em laboratório.

O experimento foi conduzido por trinta dias em delineamento inteiramente ao acaso com 8 repetições de 100 sementes cada em placas de Petri de $18 \mathrm{~cm}$ de diâmetro sobre papel de filtro umedecido na proporção de 2,5 vezes a massa. Onde o mesmo foi conduzido em germinador tipo BOD, com fotoperíodo de 12 horas e temperatura alternada (15으 no escuro e $25^{\circ} \mathrm{C}$ em luz branca).

Foram relacionados, ao longo do período de avaliação, a coloração das sementes (marrom e preta) com características do processo germinativo. Para tal, avaliou-se diariamente o número de sementes germinadas considerando-se apenas plântulas com estruturas íntegras.

Foram calculados a porcentagem de germinação (PG), o índice de sincronização ou incerteza (E) e a frequência relativa de germinação ao longo do tempo ( $f i$ ), adaptado de Labouriau (1983), bem como o coeficiente de velocidade de germinação (CVG), adaptado de Nichols \& Heydecker (1968), e o número médio de dias para germinação (MDay), segundo Edmond \& Drapala (1958).

As sementes foram classificadas quanto ao MD, conforme Ferreira et al. (2001), em sementes de germinação rápida ( $<5$ dias), intermediária ( 5 a 10 dias) e lenta ( $>10$ dias). A partir da porcentagem de germinação diária, foi construída a curva da germinação no tempo para cada classe de coloração das sementes.

$$
\begin{aligned}
& M D(\text { dias })=\left(\frac{\Sigma G i^{*} x i}{\Sigma G i}\right) \\
& \overline{\mathrm{F}} \text { (bits) }=-\sum_{\mathrm{i}=1}^{\mathrm{K}} \mathrm{fi}_{i} \log _{2} f i
\end{aligned}
$$

Onde: ni e Gi = Número de aquênios germinados no iésimo dia ou dia da observação; $\mathrm{N}$ = Número total de aquênios colocados para germinar; $\mathbf{x i}$ = Número de dias contados a partir da semeadura até o dia da observação; $\mathbf{K}=$ Ultimo dia da observação.

A massa de sementes foi avaliada, previamente ao teste de germinação, com oito amostras de 100 sementes cada. A mensuração da massa destas foi realizada em balança analítica (Brasil, 1992). Os dados foram submetidos à análise de variância, ao teste de média de Scott-Knott e à análise de correlação de Pearson, todos a 1\% de probabilidade. O software utilizado foi o Sistema de análise estatística e genética (SAEG 5.1), da Universidade Federal de Viçosa.

\section{RESULTADO E DISCUSSÃO}

Foram observadas diferenças significativas para a porcentagem de germinação (PG \%) entre as classes de sementes, em favor das de cor preta, (83\%), seguida pelas de cor marrom (46\%) (Figuras 1 e 2). Fato também observado por Ventura \& Randi (1997) em sementes de Phyllanthus niruriL. Contudo, a curva de germinação das sementes de cor preta oscilou mais que a de cor marrom (Figura 1), fato confirmado pelo índice de sincronia $\bar{E}_{\text {(bits) }}=775 \mathrm{e}$ 
$\overline{\mathrm{E}}_{\text {(bits) }}=269$, respectivamente (Figura 2).

Segundo Marcos Filho (2005), sementes de determinadas espécies, manifestam a capacidade de germinar mesmo antes de a maturidade fisiológica ser atingida; porém, em geral, são menos vigorosas por não terem alcançado o máximo acúmulo de reserva. Como observado em sementes de coloração preta, a máxima germinação ocorreu em menor número de dias $(\mathrm{MD}=23$ dias), quando comparadas com as de coloração marrom (MD= 25 dias) (Figura 2), associada a maior massa das primeiras (Figura 2). As sementes de Pothomorphe umbelata,

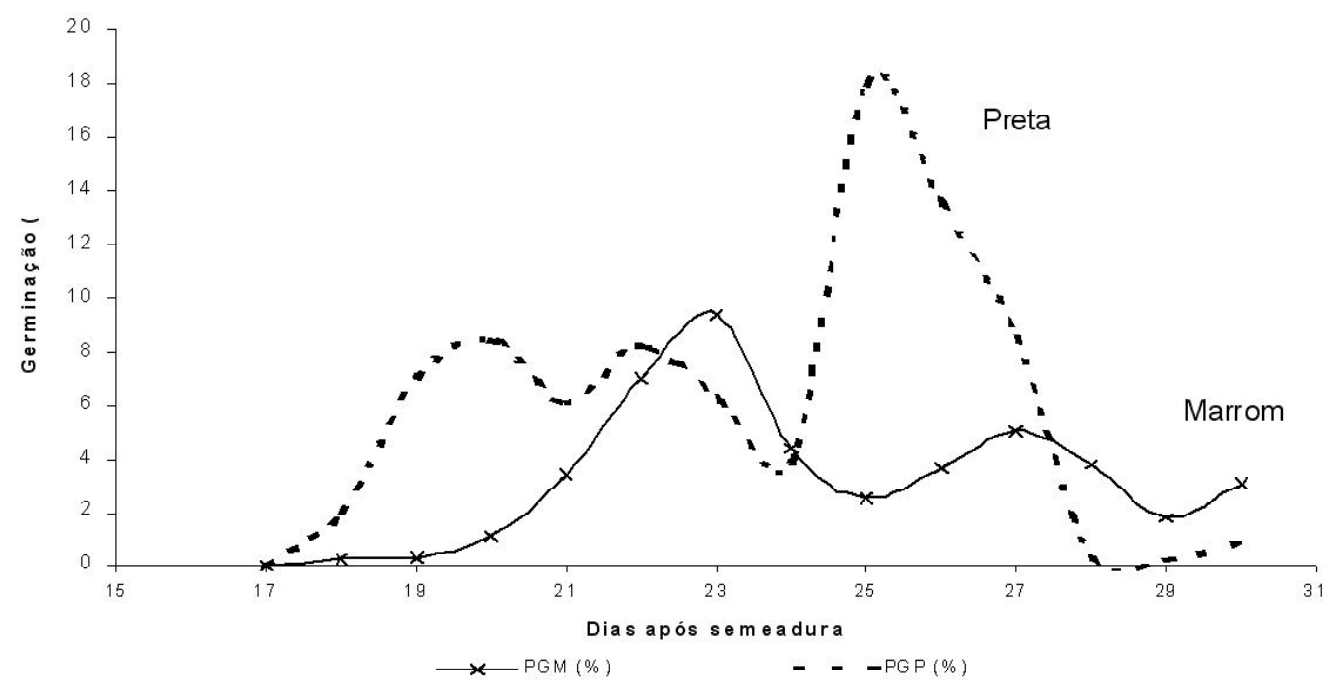

FIGURA 1. Porcentagem de germinação de sementes de Pothomorphe umbellata de colorações marrom (PGM) e preta (PGP).

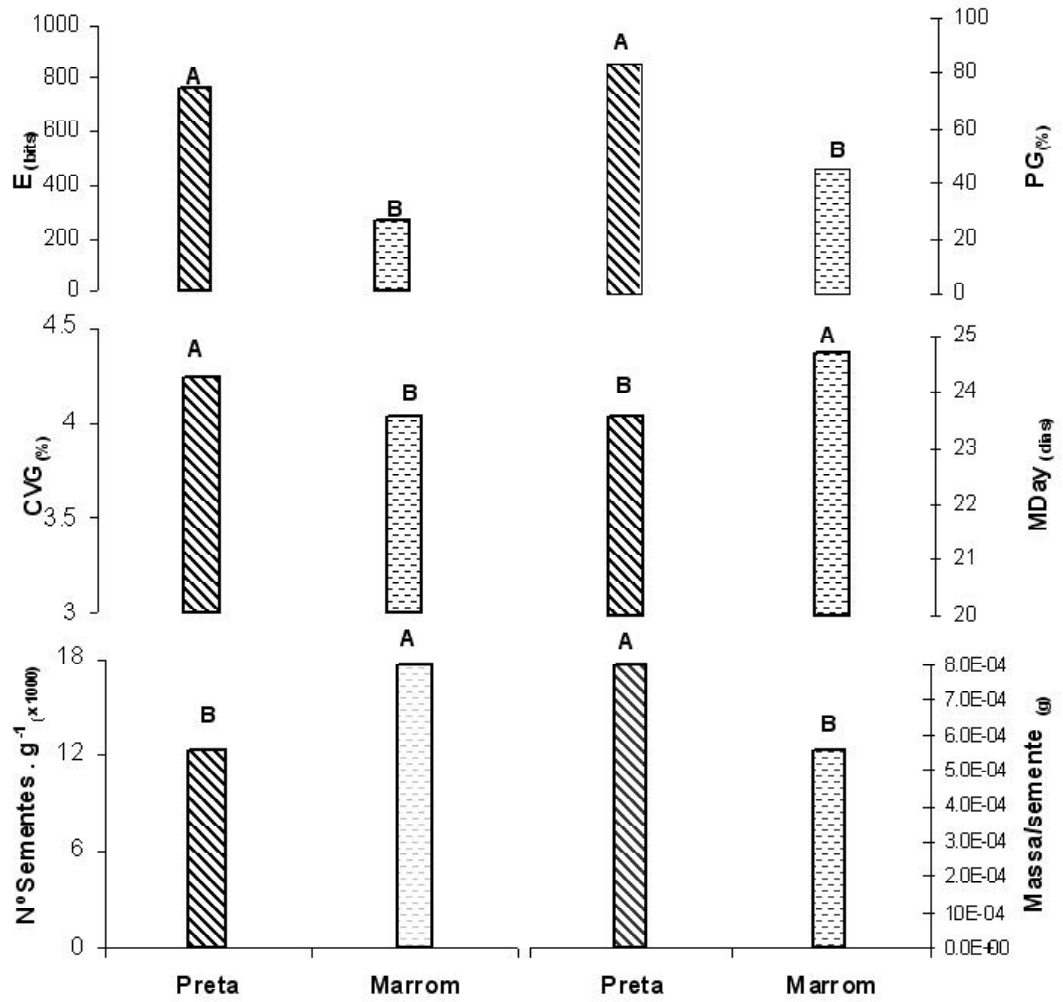

FIGURA 2. Características germinativas de Pothomorphe umbellata em função de duas classes de coloração das sementes. Onde $\mathrm{E}_{\text {(bits) }}=$ índice de sincronia; $\mathrm{PG}=$ germinação (\%); $C V G=$ Coeficiente de velocidade de germinação (\%); Mday= Número médio de dias para germinação (dias). Médias seguidas pela mesma letra maiúscula entre classes de coloração de sementes dentro de cada característica não diferem entre si pelo teste de Scott-Knott a $1 \%$ de probabilidade). 
independente da coloração, são de germinação lenta, conforme critérios indicados por Ferreira et al. (2001).

De acordo com Edmond \& Drapala (1958) e Santana \& Ranal (2004), sementes menos vigorosas demandam maior número médio de dias para germinar. No caso do coeficiente de velocidade de germinação (CVG \%), sementes com valor superior deste coeficiente são mais vigorosas, fato confirmado no presente trabalho em favor das sementes de coloração preta $(C V G=4,26)$ em relação às de coloração marrom $(C V G=4,0)$ (Figura 2 e Tabela 1). Para corroborar os resultados verificados, observouse maior massa $\left(0,8 \mathrm{mg} \mathrm{semente}^{-1}\right)$ e menor número de sementes por grama ( 12.350 sementes) para as de cor preta em relação às de cor marrom $(0,56 \mathrm{~g}$ semente $^{-1}$ e 17.800 sementes), respectivamente (Figura 2). Sementes menos pesadas refletem menor quantidade de compostos de reserva necessários ao processo de germinação, de emergência e de desenvolvimento inicial de plântulas (Marcos Filho, 2005). Portanto, as diferenças verificadas entre os índices estudados neste trabalho podem caracterizar diferenças de vigor e de porcentagem de germinação (Tabela 1) que, todavia, requerem maiores estudos.

Conforme a Tabela 1, foi observada correlação positiva entre porcentagem de germinação e massa de sementes $(r=0,91 ; p<0,01), C V G(\%)(r=0,73 ; p<$ $0,01)$ e, também, entre a $P G$ e $\circ \bar{E}_{\text {(bits) }}(r=0,98$; $\mathrm{p}<0,01)$ que foi maior em sementes de coloração preta. Por outro lado, ocorreu correlação negativa com o MD $(r=-0,73 ; p<0,01)$. Estes resultados indicam a possibilidade de haver algum tipo de impedimento físico-químico da homogeneidade de germinação que, por algum mecanismo, esteja associado à coloração da semente. A permeabilidade do tegumento à água pode variar em função do pigmento bem como da composição química (concentração de lipídios,

TABELA 1. Análises de correlação entre massa de sementes e características germinativas de Pothomorphe umbellata.

\begin{tabular}{lccccc}
\hline & $\begin{array}{c}\text { PG } \\
(\%)\end{array}$ & MES & MD & $\begin{array}{c}\text { CVG } \\
(\%)\end{array}$ & $\overline{\mathrm{E}}_{\text {(bits) }}$ \\
\hline $\mathrm{PG}_{(\%)}$ & - & 0,91 & $-0,73$ & 0,73 & 0,98 \\
$\mathrm{MES}$ & - & - & $-0,57$ & 0,58 & 0,94 \\
$\mathrm{MD}$ & - & - & - & $-0,99$ & $-0,61$ \\
$\mathrm{CVG}_{(\%)}$ & - & - & - & - & 0,61 \\
$\overline{\mathrm{E}}_{\text {(bits) }}$ & - & - & - & - & - \\
\hline
\end{tabular}

Correlações de Pearson significativas a $1 \%$ de probabilidade. $\overline{\mathrm{E}}_{\text {(bits) }}=$ índice de sincronia; $\mathrm{PG}=$ germinação (\%); $\mathrm{CVG}=$ Coeficiente de velocidade de germinação (\%); $M d=$ Número médio de dias para germinação (dias); MES= Massa/semente (g). proteínas e de compostos químicos de dormência), afetando a germinação e o desenvolvimento de plântula (Marcos Filho, 2005).

\section{CONCLUSÃO}

Considerando o tempo médio para germinação (MD) pode-se considerar que as sementes de $P$. umbelata podem ser classificadas como de germinação lenta (MD>10 dias), segundo índices de Ferreira et al. (2001), independente da coloração do tegumento (Figura 2). Segundo estes autores, tal condição caracteriza espécies de baixo potencial de ocupação de nichos, necessitando de condições muito específicas de ambiente e com baixa competição com outras espécies.

A coloração e a massa de sementes de $P$. umbellata foram características adequadas para avaliar a homogeneidade fisiológica, o vigor, potencial e comportamento germinativo. Assim, conclui-se que, embora de germinação lenta, as sementes de coloração preta associada a maior peso devem ser as escolhidas quando de coleta ou de processo seletivo para fins de propagação sexuada dessa espécie.

\section{REFERÊNCIA}

BRASIL. Ministério da Agricultura e da Reforma Agrária. Regras para análise de sementes. Brasília: SNDA/ DNDV/CLAV, 1992. 365p.

EDMOND, J.B.; DRAPALA, W.J. The effects of temperature, sand and soil, and acetone on germination of okra seed. Proceedings of the American Society for Horticultural Science, v.71 p.428-34, 1958.

FELIPPE, G.M. Qualea grandiflora: the seed and its germination. Revista Brasileira de Botânica, v.13, n.1, p.33-7, 1990.

FERREIRA, A.G. et al. Germinação de sementes de Asteraceae nativas no Rio Grande do Sul, Brasil. Acta Botânica Brasílica, v.15, n.2, p.231-42, 2001.

FRANZIN, S.M. et al. Efeito da qualidade das sementes sobre a formação de mudas de alface. Horticultura Brasileira, v.23, n.2, p.193-7, 2005.

LABOURIAU, L.G. A germinação das sementes. Washington: Programa Regional de Desenvolvimento Científico e Tecnológico, 1983. 174p.

LARCHER, W. Ecofisiologia vegetal. Rima: São Carlos, 2000. 531p.

MALUF, A.M.; WIZENTIER, B. Aspectos fenológicos e germinação de sementes de quatro populações de Eupatorium vauthierianum DC. (Asteraceae). Revista Brasileira de Botânica, v.21, n.3, p.19-26, 1998.

MARCOS FILHO, J. Fisiologia de sementes de plantas cultivadas. Piracicaba: FEALQ, 2005. 495p.

MARCOS FILHO, J. New aproaches to seed vigor testing. Scientia Agricola, v.55, n.esp., p.27-33, 1998.

MARCOS FILHO, J. Testes de vigor: importância e utilização. In: KRZYZANOWSKI, F.C.; VIEIRA, R.D.; FRANÇA NETO, J.B. Vigor de sementes: conceitos e

Rev. Bras. PI. Med., Botucatu, v.13, n.1, p.116-120, 2011. 
testes. Londrina: ABRATES, Comitê de Vigor de Sementes, 1999. 218p.

NICHOLS, M.A.; HEYDECKER, W. Two approaches to the study of germination data. Proceedings of the International Seed Testing Association, n.33, v.3, p.53140, 1968.

PIANA, Z.; TILLMANN, M.A.A.; MINAMI, K. Avaliação fisiológica de sementes de cebola e sua relação com a produção de mudas vigorosas. Revista Brasileira de Sementes, v.17, n.2, p.149-53, 1995.

RODO, A.B. Avaliação do potencial fisiológico de sementes de cebola e sua relação com o desempenho das plantas em campo. 2002. 123p. Tese (Doutorado Fitotecnia) - ESALQ, USP, Piracicaba.

ROSSETTO, C.A.V. et al. Efeito da disponibilidade hídrica do substrato, da qualidade fisiológica e do teor de água inicial das sementes de soja no processo de germinação. Scientia Agricola, v.54, n.1-2, p.97-105, 1997.
ROSSETTO, C.A.V.; VIEGAS, E.C.; NAKAGAWA, J. Germinação das unidades de dispersão de gervão-roxo. Horticultura Brasileira, v.18, n.2, p.114-8, 2000. SANTANA, D.G.; RANAL, M.A. Analise de germinação: um enfoque estatístico. Brasília: Ed. Universidade de Brasília, 2004. 248p.

SANTOS, S.G.R.; AGUIAR, I.B. Efeito da temperatura na germinação de sementes de Sebastiana commersoniana (Baillon) Smith \& Downs separadas pela coloração de tegumento. Scientia Florestalis, v.69, p.77-83, 2005.

UNANDER, D.W. et al. Factors affeting germination and stand establishment of Phyllanthus amarus (Euphorbiaceae). Economic Botany, v.49, n.1, p.49-55, 1995.

VENTURA, S.; RANDI, A.M. Influência da coloração das sementes na germinação de Phyllanthus tenellus Roxb. e Phyllanthus niruri L. (Euphorbiaceae). Acta Botânica Brasílica, v.11, n.1, p.87-94, 1997. 